\title{
ENCAPSULATION OF IBUPROFEN INTO SOLID LIPID NANOPARTICLES FOR CONTROLLED AND SUSTAINED RELEASE USING EMULSIFICATION SOLVENT EVAPORATION TECHNIQUE
}

\author{
WESLEY N OMWOYO, MAKWENA J MOLOTO* \\ Department of Chemistry, Vaal University of Technology, Private Bag X021, Vanderbijlpark, South Africa. Email: makwenam@vut.ac.za \\ Received: 18 April 2019; Revised and Accepted: 12 July 2019
}

ABSTRACT

Objective: The objective of the study was to encapsulate ibuprofen (IBU) into solid lipid nanoparticles (SLNs) for enhanced dissolution and achieving a sustained and controlled release of the drug from the nanocarrier.

Methods: IBU loaded nanoparticles were prepared by emulsification solvent evaporation technique and characterized by Fourier Transform Infrared spectroscopy, Thermogravimetric Analysis, X-ray diffraction (XRD), and transmission electron microscopy. Release kinetics on the drug-loaded nanoparticles was carried out in phosphate buffer $\mathrm{pH} 6.8$ using pharma test dissolution apparatus adopting shaking basket method at $37^{\circ} \mathrm{C}$.

Results: The optimized IBU-loaded SLNs had a particle size of $76.40 \mathrm{~nm}$, polydispersity index of 0.275 , and zeta potential of $-41.3 \mathrm{mV}$. The encapsulation efficiency (EE) and DL were $99.73 \%$ and $2.31 \%$, respectively. The Fourier transform infrared spectroscopy (FTIR) spectra confirmed successful encapsulation of the drug inside the nanocarrier as only peaks responsible for the emulsifier and the binder could be identified. This corroborated well with XRD spectra which showed a completely amorphous state of the drug-loaded nanoparticles as compared to the crystalline nature of the pure drug. The IBU-SLNs showed a release profile of up to $8 \mathrm{~h}$ which is a great improvement from other reported works. The drug release pattern of IBU-SLNs was best fitted with Higuchi square root model and followed the Higuchi drug release kinetics. Korsmeyer-Peppas model confirmed a non-Fickian diffusion model for the release of the drug from the matrix system.

Conclusion: IBU-loaded SLNs were successfully prepared which had a sustained and controlled release. It was observed that the release of the drug from the matrix was diffusion controlled and time dependent.

Keywords: Cyclooxygenase, Nociceptors, Inflammation, Ibuprofen, Nanomedicine.

(C) 2019 The Authors. Published by Innovare Academic Sciences Pvt Ltd. This is an open access article under the CC BY license (http://creativecommons. org/licenses/by/4. 0/) DOI: http://dx.doi.org/10.22159/ajpcr.2019.v12i7.33652

\section{INTRODUCTION}

Ibuprofen (IBU), a phyl propionic acid derivative is widely used as a first-line nonsteroidal anti-inflammatory drug (NSAID) which is reported to have short half-life and is poorly water-soluble [1]. This drug is normally used to reduce or clear pain though it is also effective in the treatment of rheumatoid arthritis, osteoarthritis, and inflammations. When the body cells are damaged, they release arachidonic acid which is broken down by cyclooxygenase (Cox), Cox 1 and Cox 2 enzymes to form prostaglandin $\mathrm{H}_{2}$ which is, in turn, converted into multiple chemicals. These chemicals are responsible for raising the body temperature, cause inflammations, and lower the body pain threshold [1,2]. The lowering of the body pain threshold causes the nociceptors to send a signal to the spinal cord, which in turn relays it to the brain to alert it of the pain. When IBU is taken, it is absorbed in the bloodstream through the stomach walls. The IBU then travels and attaches itself to the two enzymes Cox 1 and Cox 2 which creates a barrier between the enzymes and arachidonic acid and thus preventing the formation of prostaglandin $\mathrm{H}_{2}$ which normally lowers the body pain threshold [2]. By so doing the information of the pain will not be relayed to the brain. The IBU undergoes efflux after some short while, and the circle continues (Fig. 1)

Although IBU has poor water solubility [3], it is absorbed when orally given to give a maximum plasma concentration around $1-2 \mathrm{~h}$ after ingestion $[4,5]$. It is, however, rapidly eliminated from systemic circulation due to its short half-life, thus requiring several dosages for it to be effective [6]. The drug can be taken for chronic diseases and the maintenance of pharmacological activity is more important than how fast the drug acts. The frequent usage of this drug has led to increased side effects due to the high doses used and non-compliance of patients. IBU is still the drug of choice as compared to other NSAIDs [7], though it has demonstrated drug-related gastric toxicity, such as gastric irritation, bleeding, abdominal pain, and ulcers [8-10]. Nanotechnology has revolutionized the field of drug delivery system. Research has proven that nanoparticles can be prepared as effective tools for drug delivery. The nanoparticles can overcome the limitation of drug delivery by penetrating the physiological barrier to deliver the drug to the site of action or absorption $[2,3]$. The nanocarriers aid in the extension of the residence time in the small intestines to ensure maximum absorption of the drug which in turn increases the therapeutic activity $[3,4]$. With nanotechnology, the drug can either be conjugated on the surface of the prepared nanoparticle or encapsulated and protected inside the drug carrier matrix [2-4]. Nanocarriers have previously been prepared from a wide range of polymers or fatty acids. Biodegradable polymers or fatty acids are usually used to encapsulate drugs for short-term therapy, while non-biodegradable polymers are used to encapsulate drugs for long-term therapy such as the administration of vaccines and hormones [7]. IBU is known to be a purely crystalline drug, which is the main reason for its poor water solubility. The physical transformation of a crystalline drug into a more soluble but meta-stable amorphous form will be of the essence in improving the delivery of IBU drug $[11,12]$. Selection of a matrix is essential in the preparation of nanocarriers since it affects particle size and release kinetics. Surfactants or stabilizers have been also reported to have greater influence in the formation of nanocarriers for drug delivery $[13,14]$. They are known to decrease the interfacial tension between lipophilic and hydrophilic phases of the emulsion and ease the formation of the drug carriers by enhancing their stability [15]. Encapsulation of drugs into fatty acids using a suitable surfactant has been reported to increase solubility and oral absorption 
of poorly water-soluble drugs [16-18]. This encapsulation is what leads to the formation of the solid lipid nanoparticles (SLNs) which are submicron colloidal drug carriers composed of lipids or waxes dispersed in an aqueous surfactant solution $[17,19,20]$.

The current study aims to encapsulate IBU drug into SLNs using stearic acid (SA) as a carrier matrix and polyvinylpyrrolidone (PVP) as a surfactant. The effect of organic to aqueous ratio was also determined in relation to particle size and size distribution. The optimized formulation was then characterized to confirm particle properties. Further, kinetic studies were performed on the in vitro release data to help explain the drug release mathematically.

\section{MATERIALS AND METHODS}

Materials

The SA of molecular weight $284.48 \mathrm{~g} / \mathrm{mol}$, PVP of molecular weight 13,000-23,000, D-lactose monohydrate (DLM), molecular weight $360.312 \mathrm{~g} / \mathrm{mol}$, and ethyl acetate (EtoAc) were used in the current study. All other chemicals used were of analytical grade and were purchased from Sigma-Aldrich, Johannesburg, South Africa. In this experimental design, SA was the matrix; PVP was used as an emulsifier and/or surfactant, and lactose was used as a binder.

\section{Preparation of IBU loaded SLNs}

The nanoparticle drug carriers were prepared by the method described by Omwoyo et al., $[17,19]$ with slight modifications. Nanoparticle drug carriers for IBU were prepared using a modified emulsification solvent evaporation technique. The organic phase was prepared by dissolving SA and IBU in EtoAc under stirring with a magnetic stirrer. The aqueous surfactant solution constituted of 5\% (w/v) PVP and 5\% (w/v) DLM. The organic phase was dispersed in the aqueous phase by means of a high-speed homogenizer (Silverson L4R, Silverson Machines Limited, Buckinghamshire, United Kingdom) with a speed of $6000 \mathrm{rpm}$ for $5 \mathrm{~min}$. The resulting oil-in-water single emulsion $(\mathrm{O} / \mathrm{W})$ obtained was directly fed into a bench top Buchi Mini Spray Dryer (Model B-290, BÜCHI Labortechnik AG, Flawil, Switzerland) and spray-dried at $110^{\circ} \mathrm{C}$, with an atomizing pressure of 5 bars, $100 \%$ aspirator rate, and $20 \%$ flow rate. This was done to four different formulations, as shown in the experimental design (Table 1). The optimized formulation was then fully characterized and release kinetics studied.

\section{Characterization of the drug-loaded nanoparticles}

Particle size analysis and polydispersity index (PDI)

Particle size analysis of the produced nanocarriers was determined by dynamic light scattering photon correlation spectroscopy (PCS) using a Malvern Zetasizer Nano ZS (Malvern Instruments, Worcester shire, United Kingdom). The PCS yielded the mean diameter and the PDI which is a measure of the width of the size distribution. The mean diameter and PDI values were obtained at an angle of $90^{\circ}$ in $10 \mathrm{~mm}$ diameter cells at $25^{\circ} \mathrm{C}$. Before the measurements of each sample, approximately 1-3 mg of the nanoparticles were suspended in distilled water, then vortexed and sonicated for a few minutes to produce a suitable scattering intensity.

\section{Zeta potential}

The zeta potential, reflecting the electric charge on the particle surface and indicating the physical stability of colloidal systems, was determined by electrophoretic mobility using the Malvern Zetasizer Nano ZS (Malvern Instruments, Worcester shire, United Kingdom). The sample was prepared as for particle size measurement but a different curvet that could conduct current during the measurement.

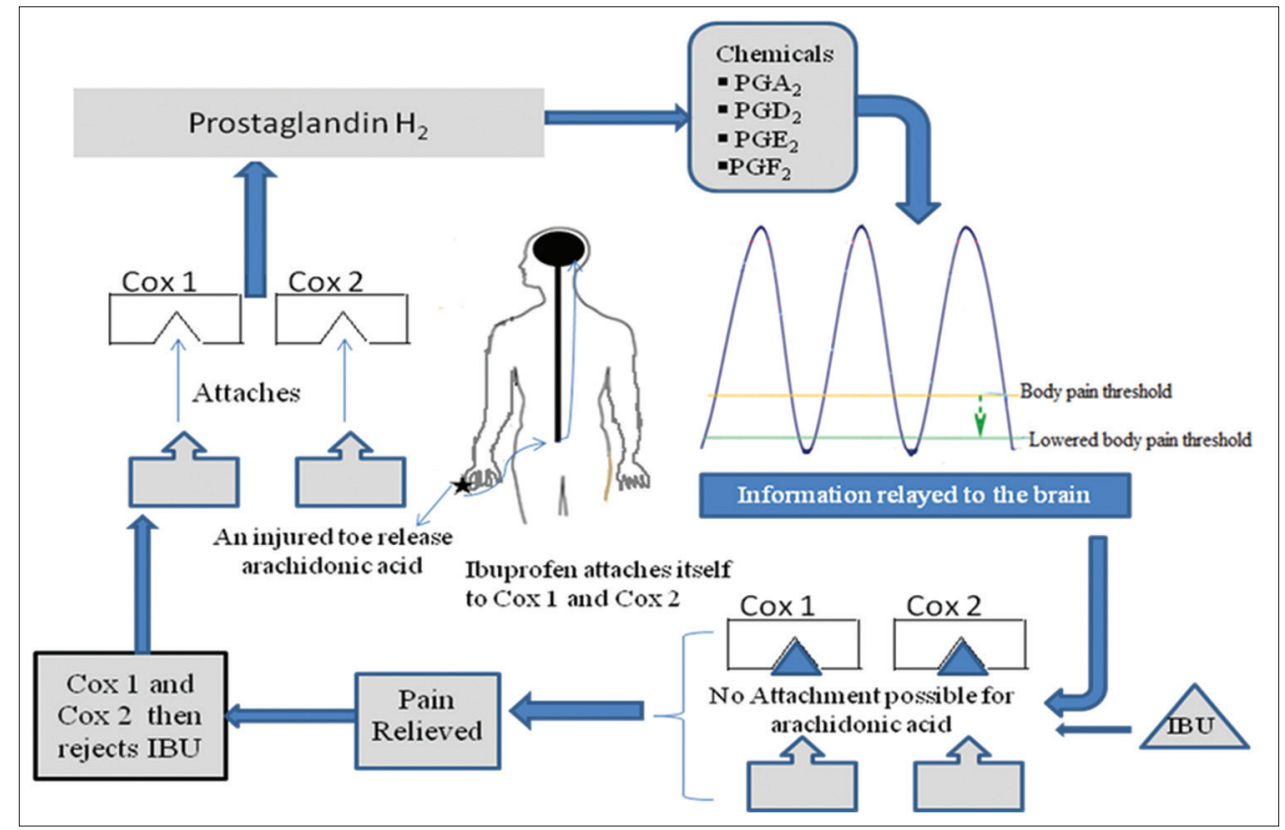

Fig. 1: Schematic demonstration on how ibuprofen relieves pain

Table 1: Experimental design of preparing IBU-loaded nanoparticles

\begin{tabular}{|c|c|c|c|c|c|c|}
\hline \multirow[t]{2}{*}{ Formulation } & \multicolumn{3}{|c|}{ Internal phase (I) } & \multicolumn{2}{|c|}{ External phase (E) } & \multirow[t]{2}{*}{ Ratio (I: E) } \\
\hline & SA (mg) & EtoAc $(\mathrm{mL})$ & IBU (mg) & PVP (mL) & DLM (mL) & \\
\hline F1 & 50 & 10 & 50 & 7 & 3 & $1: 1$ \\
\hline F2 & 50 & 10 & 50 & 20 & 10 & $1: 3$ \\
\hline F3 & 50 & 10 & 50 & 40 & 20 & $1: 6$ \\
\hline Placebo & 50 & 10 & - & 40 & 20 & $1: 6$ \\
\hline
\end{tabular}

SA: Stearic acid, EtoAc: Ethyl acetate, IBU: Ibuprofen, PVP: Polyvinylpyrrolidone, DLM: D-lactose monohydrate 
Determination of EE and DL

The EE was determined using the indirect method [17]. In brief; $20 \mathrm{mg}$ of the prepared sample of nanoparticles were dispersed in $10 \mathrm{~mL}$ of water and vortexed for $3 \mathrm{~min}$. The resultant solution was then ultracentrifuged at $15,000 \mathrm{rpm}$ for $20 \mathrm{~min}$ at $4^{\circ} \mathrm{C}$. The supernatant was discarded as a means of "washing" the nanoparticles. The precipitate in the falcon tubes was then dissolved in $10 \mathrm{~mL}$ of phosphate buffer saline $\mathrm{pH} 7.2$ by vortex and sonication. This sample was then analyzed in ultraviolet (U.V) spectrophotometer (T80+, UV/Vis spectrometer, PG Instruments Ltd.) at $221 \mathrm{~nm}$. The EE\% and DL\% were calculated using the formulas below:

- $\quad \mathrm{EE} \%=($ Drug in precipitate*/total added drug $) \times 100$

- $\mathrm{DL} \%=\left(\right.$ Drug in precipitate/total drug+added excipients $\left.{ }^{* *}\right) \times 100$

- $\quad$ *Drug in precipitate=Total drug added - free drug after ultracentrifugation

- **Added excipients=Lipids+surfactant+binder

\section{Thermal properties}

Thermogravimetric analysis (TGA) was used to measure the physical and chemical changes of the drug and drug-loaded nanoparticles as a function of temperature. A sample of IBU-free drug, placebo nanoparticles and IBU-loaded nanoparticles were analyzed using TGA (TGA-4000; PerkinElmer, Thermogravimetric analyzer, USA) connected to an inert nitrogen gas flow and at a heating rate of $10^{\circ} \mathrm{C} / \mathrm{min}$. TGA was also used to confirm the approximate DL capacity.

\section{Surface morphology}

Transmission electron microscopy (TEM) was used to provide a way to directly observe the morphological appearance of the nanoparticles and the shape. The placebo and drug-loaded nanoparticles were placed on a formvar-coated grid; air dried then loaded on the TEM, JEOL 1400, for image collection. Fourier transform infrared spectroscopy (FTIR) was also used to determine the functional groups present on the surface of the nanoparticle. This was done on FTIR, Nicolet, iS50, FT-IR spectrometer.

\section{$X$-ray diffraction (XRD) studies}

$X$-ray powder diffraction studies of pure IBU drug and the IBU-loaded nanoparticles were performed using Siemens D500. Measurement conditions included target, $\mathrm{CuK} \alpha$, voltage, $40 \mathrm{kV}$ and current, $30 \mathrm{~mA}$. A system of diverging, receiving, and anti-scattering slits of $1^{\circ}, 1^{\circ}$, and $1^{\circ}, 0.15^{\circ}$, respectively, were used. Jade 6.0 will was used for data collection and processing (Materials Delta Inc. U.S.A.).

\section{In vitro release experiment}

In vitro drug dissolution studies were performed for optimum formulations. Pharma Test Dissolution Apparatus (D 63514, Hainburg Germany) was used for the in vitro IBU release studies. This method adopted a rotating basket method which was filled with water, and the temperature was maintained at a constant value of $37 \pm 0.1^{\circ} \mathrm{C}$. The rotating speed of the basket was kept constant at $100 \mathrm{rpm}$. The dissolution solvent used was phosphate buffer saline $\mathrm{pH} 6.8$ where $20 \mathrm{mg}$ of IBU-loaded nanoparticles were put in $10 \mathrm{~mL}$ of the solvent in falcon tubes. The falcon tubes were then placed in the shaking basket and the drug diffusion into the solvent allowed to take place over time. At specific time intervals, samples were withdrawn from the dissolution solvent and immediately replaced by a fresh solvent which was already stored at the same temperature. The samples were analyzed using UV-Vis spectrophotometer at $221 \mathrm{~nm}$ to get their absorbance and subsequent concentrations of the drug dissolved in the solvent. The in vitro drug release was then plotted as cumulative drug released versus time to indicate how long the formulation released. Furthermore, the drug release kinetics from the matrix of each of the formulations was subjected to zero-order kinetics, first-order kinetics, Higuchi kinetics, and Korsmeyer-Peppas models for further interpretations.

\section{RESULTS AND DISCUSSION}

\section{Particle size and PDI}

The particle size measurements, polydispersity, zeta potential, EE, DL, and yield for formulation F1, F2, F3, and the placebo are given in Table 2. The placebo nanoparticles were prepared after the optimal formulation was identified. The PCS graphs yielded from a dynamic light scattering of the zetasizer are presented in Fig. 2 to confirm the particle sizes and PDI.

From the results obtained, the formulation with an internal to the external ratio of 1:1 comparatively gave the largest particle sizes with a non-uniform particle distribution (PDI=0.304). The PDI values are expected to be generally low (0.001-0.290) for a homogeneous system [19]. The higher values of PDI are an indication of a very wide distribution of particle sizes, and this is also evident from the nature of the graph obtained from the PCS analysis (Fig. 2).

Similar results were obtained when primaquine was encapsulated into SLNs containing SA in organic internal phase and aqueous surfactants of polyvinyl alcohol and lactose in the external phase. A ratio of 1:1 gave particle sizes of $653.7 \pm 8.7 \mathrm{~nm}$ and a PDI of $0.61 \pm 0.12$ [17]. Equally, when IBU was encapsulated to PVP alone with a ratio of $1: 1$, the particle sizes were $670 \pm 76.39 \mathrm{~nm}$ and a PDI of 0.65 [12]. This observation was attributed to the difficulty in dispersing one phase into the other phase as the same quantities were used. In an ideal scenario, the dispersed phase should be of smaller quantities than the dispersant $[12,17]$. Formulations F2, F3, and the placebo showed particle sizes of $81.62 \mathrm{~nm}$, $76.40 \mathrm{~nm}$, and $70.58 \mathrm{~nm}$, respectively. The placebo nanoparticles were slightly smaller in size compared to the drug-loaded nanoparticles. The PDI of the three formulations was $0.279,0.275$, and 0.257 , respectively, and this shows an acceptable particle size distribution, which is homogeneous and monodispersed [12].

\section{Zeta potential}

Zeta potential is used to determine the physical stability of nanoparticles. It is a measure of particle surface charge and an indirect measurement of the thickness of the diffusion layer [12]. It is usually used as an indicator to predict long-term stability of pharmaceutical formulations [18]. High values of zeta potential correlate with a lesser tendency to aggregate or flocculate and are indicative colloidal stability of the nanoformulation [21]. Studies have reported that electrostatically stable nanoformulations should have a minimum zeta potential of $\pm 30[10,19]$. It is evident from the results given in Table 2 that all the prepared formulations showed good stability as seen from the high values of zeta potential. This was expected as similar other researches using PVP as an emulsifier reported high values of negative zeta potential [12]. Nada et al. prepared several formulations of IBU loaded nanoparticles and nanosuspensions using PVP as the emulsifier and obtained zeta potential values of between $39.2+0.976$ and $58.9+0.503[12]$.

Table 2: Preliminary characterization of loaded and unloaded nanoparticles

\begin{tabular}{lllllll}
\hline Formulation & PS $(\mathbf{n m})$ & PDI $^{*}$ & ZP $(\mathbf{m V})$ & EE (\%) & DL (\%) & Yield (\%) \\
\hline F1 & 136.40 & 0.304 & -36.8 & - & - & - \\
F2 & 81.62 & 0.279 & -43.6 & 93.98 & 4.01 & $(1173 \mathrm{mg}) 73.31$ \\
F3 & 76.40 & 0.275 & -41.3 & 99.73 & 2.31 & $(2153 \mathrm{mg}) 69.45$ \\
Placebo & 70.58 & 0.257 & -42.5 & - & - & - \\
\hline
\end{tabular}

PS: Particle size, PDI: Polydispersity index, ZP: Zeta potential, EE: Encapsulation efficiency, DL: Drug loading 


\section{EE and DL}

EE is the percentage of the drug entrapped inside the nanocarrier relative to the total amount of drug added to the formulation. On the other hand, DL is defined as the percentage of the drug entrapped in relation to the total amount of drug added and all other excipients [22]. A good formulation should strike a balance between EE and DL. There should be high EE and a relatively high DL capacity. From the data shown in Table 2, in formulation F2, approximately $6 \%$ of the IBU was detected by UV-vis as un-encapsulated and thus the $93.98 \% \mathrm{EE}$ while for the F3 formulation the UV-vis could only detect traces of IBU corresponding to $<1 \%$ and thus the $99.73 \%$ EE. This could be attributed to the increase of the external phase in F3 which gives room for complete encapsulation of the drug substance. The two formulations generally exhibited high EE and this can be attributed to the poor solubility of IBU in PVP. Previous studies have shown the effectiveness of using water-soluble surfactants for formulating small size nanoparticles with high entrapment efficiency for poorly water-soluble drugs [23]. The DL capacity of F2 and F3 was $4.01 \%$ and $3.21 \%$, respectively. These values were expected as the amounts of excipients added were much more compared to the drug in the formulations.

\section{FTIR analysis}

FTIR spectroscopy was used to obtain conformational information about the IBU loaded SLNs. The interaction between IBU and SA which is the carrier matrix and the surfactant was monitored by the use of the FTIR (Fig. 3). The intense peaks appearing in the spectra of IBU and PVP are due to the asymmetric stretching vibrations of their functional groups. The spectrum of IBU shows a well-defined infrared band around $1680-1750 \mathrm{~cm}^{-1}$, which is the characteristic peak of the carbonyl stretching of the isopropanoic acid group. There are also peaks between 2900 and $3000 \mathrm{~cm}^{-1}$ and around 3000 and $3100 \mathrm{~cm}^{-1}$ which are characteristic peaks for carboxylic acid, $\mathrm{O}-\mathrm{H}$ stretching and aromatic C-H stretching, respectively. The peak around $1500 \mathrm{~cm}^{-1}$ is synonymous with the aromatic C-C stretching. The IR spectrum of PVP shows characteristic peaks around $1650 \mathrm{~cm}^{-1}$ for the carbonyl stretching, around $1400 \mathrm{~cm}^{-1}$ for the $\mathrm{C}-\mathrm{H}$ alkane bend, and around $2900 \mathrm{~cm}^{-1}$ for the C-H alkane stretch ( $\mathrm{Sp}^{3}$ hybridized carbon). The characteristic peak seen on the nanoparticles around 1000-1100 $\mathrm{cm}^{-1}$ is the $\mathrm{C}-\mathrm{O}$ bond from lactose. The spectra of the placebo nanoparticles and drug loaded nanoparticles show similar characteristic peaks of the surfactant used and partly of the binder though with very slight modifications. The nanoparticles show a slight shift of the carbonyl peak around $1650 \mathrm{~cm}^{-1}$. There is also a slight shift and broadening of the peak around $2800-3000 \mathrm{~cm}^{-1}$. The shifts and broadening of the peaks are indicative of possible hydrogen bonding between IBU, PVP which is the surfactant and lactose the binder. The decrease in intensity and shifts is also visible in the peaks around $1400 \mathrm{~cm}^{-1}$, suggesting that this group is taking part in the hydrogen bonding process. The chemical shifts could also be attributed to physical interactions of the drug and the surfactant together with the binder, which in turn enhanced aqueous solubility, wettability, and drug dissolution [12].

The spectra of the drug, however, did not show any new peaks, indicating that there was no new chemical bond formed between the drug and the excipients [24]. The characteristic peaks unique to the drug were also not observed on the prepared drug-loaded SLNs indicating successful encapsulation of the drug into the nanocarrier. This FTIR data showed that molecular interactions that could alter the chemical structure of the IBU did not occur. It can, therefore, be noted that there is no chemical interaction between the functional groups of the drug and the added excipients [24]

\section{XRD}

The pure IBU drug and drug-loaded nanoparticles were subjected to X-ray power diffractions and the diffractograms compared. The characteristic intensity peaks for pure IBU could be identified around $12.0^{\circ}, 16.0^{\circ}, 17.0^{\circ}, 22.0^{\circ}, 25.0^{\circ}, 27.0^{\circ}, 30.0^{\circ}$, and $34.0^{\circ} 2 \theta$ degrees which is in agreement with literature $[18,24]$. In the drug-loaded nanoparticles, these peaks are seen to disappear (Fig. 4) which indicates the existence of an amorphous state of the nanoparticles as compared to the crystalline nature of the drug. This is attributed to the incorporation of IBU into the lipid matrix and successful encapsulation of the drug inside the excipients $[18,19,25]$.

Previous studies have attributed the reduced intensity to the presence of IBU in the crystal lattice which changes the crystallinity of the SLNs [25]. When IBU is encapsulated only in a lipid matrix, there is the disappearance of several IBU peaks which usually represents the coexistence of the amorphous and crystalline structure within the lipid matrix [18]. Akbari et al. [24] also prepared IBU-loaded SA nanoparticles and the intensity peaks could be identified at $21.28,19.8$, 16.32 , and 23.44 which clearly showed that in the diffractograms the high intensity peaks belonged to the lipid and thus the disappearance of the IBU peaks signified the coexistence of amorphous and crystalline
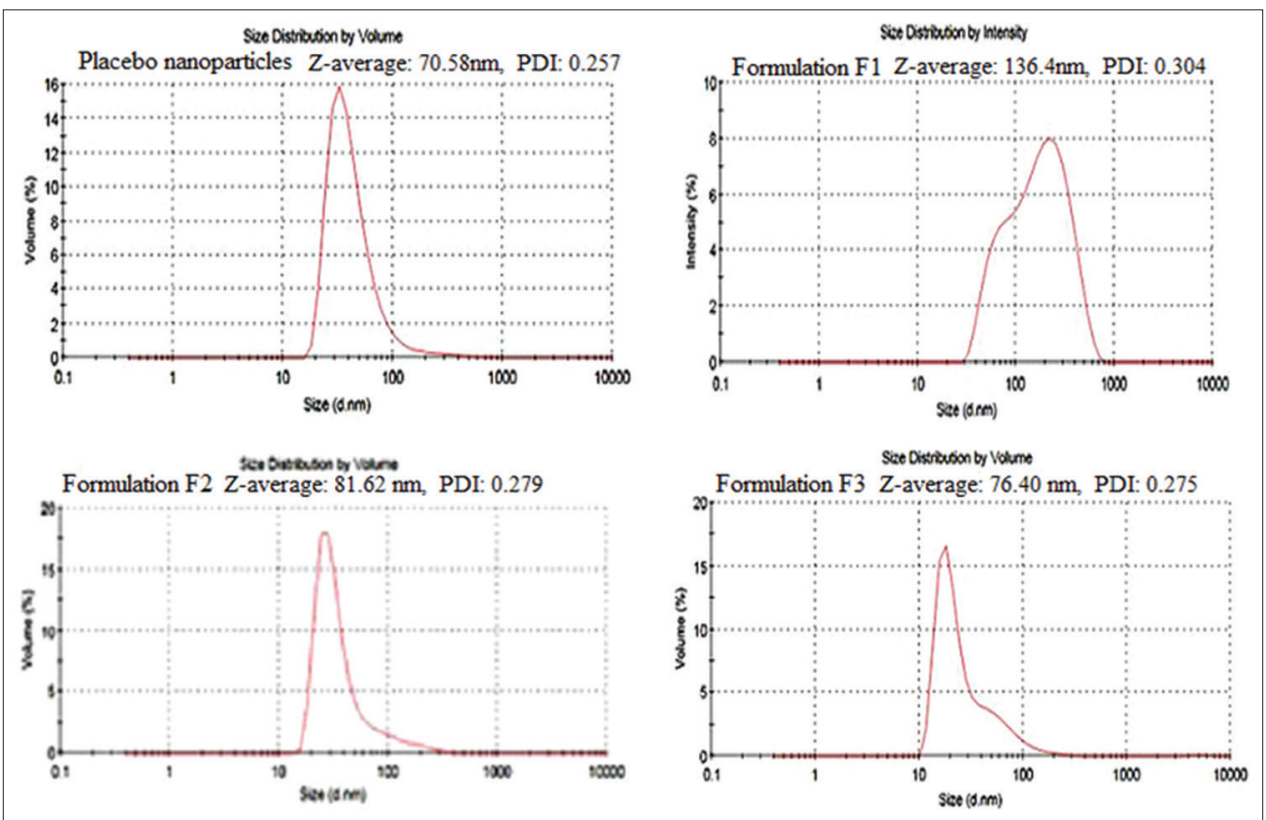

Fig. 2: Photon correlation spectroscopy graphs for particle size measurements and PDI for placebo nanoparticles, formulations F1, F2, and F3 


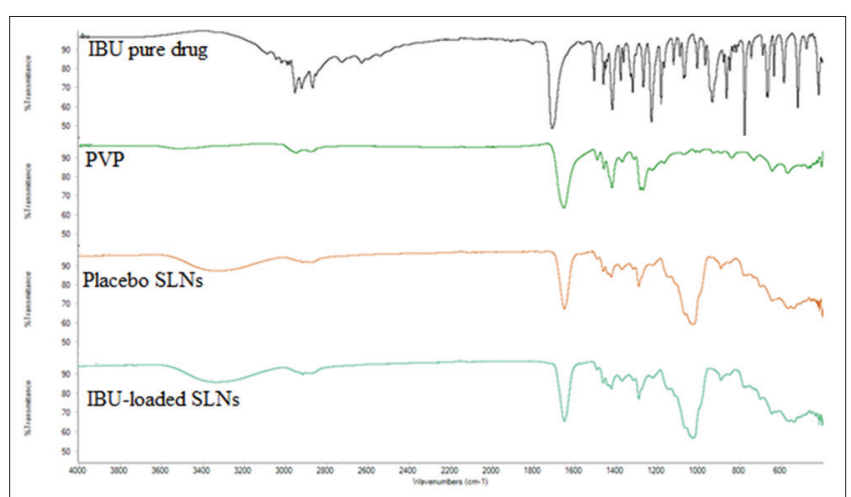

Fig. 3: Fourier transform infrared spectroscopy spectra of pure ibuprofen, polyvinylpyrrolidone, placebo nanoparticles, and IBUloaded nanoparticles

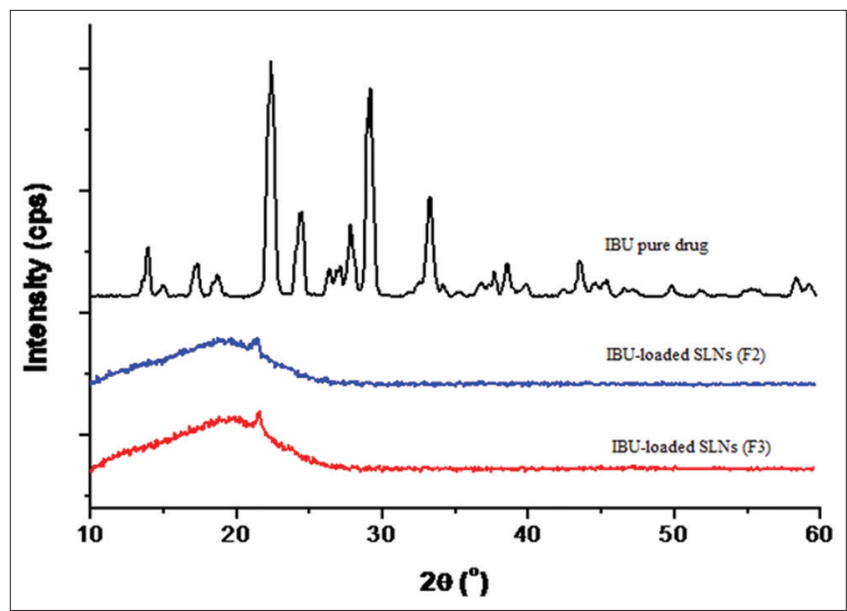

Fig. 4: X-ray diffraction diffractograms of pure ibuprofen (IBU) drug and IBU-loaded solid lipid nanoparticles

IBU in the lipid matrix. The addition of surfactants and other excipients are known to further lessen the peak intensities or make them not visible and thus modify the crystallinity of the drug leading to a more amorphous nature of the formulation $[10,26]$. Similar results were obtained by Hasnain and Kumar [26], where the pure IBU exhibited a completely crystalline nature, the physical mixture of IBU with PEG 6000 and PVP-K30 exhibited a coexistence of amorphous and crystalline nature while the IBU solid dispersions using PEG 600-PVP $\mathrm{K} 30$ as a combined carrier exhibited a completely amorphous state as recorded from the XRD [26].

\section{Thermogravimetric analysis}

The TGA thermograms for IBU, placebo nanoparticles, and IBU-loaded nanoparticles are presented in Fig. 5. For the placebo and drug-loaded nanoparticles, three steps of weight loss were observed. The first covered the temperature range of about $40-100^{\circ} \mathrm{C}$, corresponding to the loss of moisture. From the thermogram, this range exhibited a weight loss of about $5 \%$, which is acceptable for pharmaceutical products [19]. The second and the third covered the temperature ranges of about $220-300^{\circ} \mathrm{C}$ and about $240-450^{\circ} \mathrm{C}$ corresponding to the thermal degradation of PVP and lactose. With regard to the TGA thermogram of IBU, it exhibited one major step of weight loss covering the temperature ranges of about $180-250^{\circ} \mathrm{C}$. It is also documented that the difference in weight percent between the drug-loaded nanoparticles and the placebo nanoparticles can give an approximate indication of the DL percentage [27]. In this study, the current TGA thermogram (Fig. 5) indicates a DL of approximately 3-6\%, which is similar to the calculated value of 2.31 and $4.01 \%$ for the formulations. Several factors determine the loading capacity of the drug in the nanoparticles.
These factors include the solubility of the drug in the lipid matrix, physicochemical properties of the matrix used, and the miscibility of the drug, and the matrix used [17]. Addition of stabilizers or emulsifiers has previously been reported to increase the EE and lowered DL capacity $[17,19]$. In the current study, the low DL capacity is attributed to the high amounts of the surfactant and binder used, and this is the reason behind the high EE and longer release profile.

\section{TEM}

The TEM images of the prepared IBU-loaded and unloaded nanoparticles are presented in Fig. 6. In general, TEM showed the particles to be spherical in shape. The placebo nanoparticles were generally small and well dispersed in relation to the IBU-loaded nanoprticles. The IBU-loaded nanoparticles were larger in size signifying successful encapsulation of the drug into the nanocarrier. This observation indicates that loading actually took place. These results are in line with the results obtained from zeta sizer and thermogravimetric analysis.

\section{In vitro release studies}

The two drug-loaded formulations F2 and F3 both having SA as a carrier matrix, PVP as a surfactant and lactose as a binder were found to be able to sustain the drug release (Fig. 7). The cumulative percent drug release for the two formulations was found to be $94 \%$ and $96 \%$ at the end of about $7 \mathrm{~h}$ and $8 \mathrm{~h}$, respectively. Both formulations did not show an initial burst release indicating a complete encapsulation in the carrier matrix and lack of unbound free drug concentrations on the surface of the nanoparticles [28].

This collaborated well with the FTIR spectral data that did not show any peak responsible for the drug. It was observed that F3 formulation was able to sustain the drug release a little longer, and this was attributed to

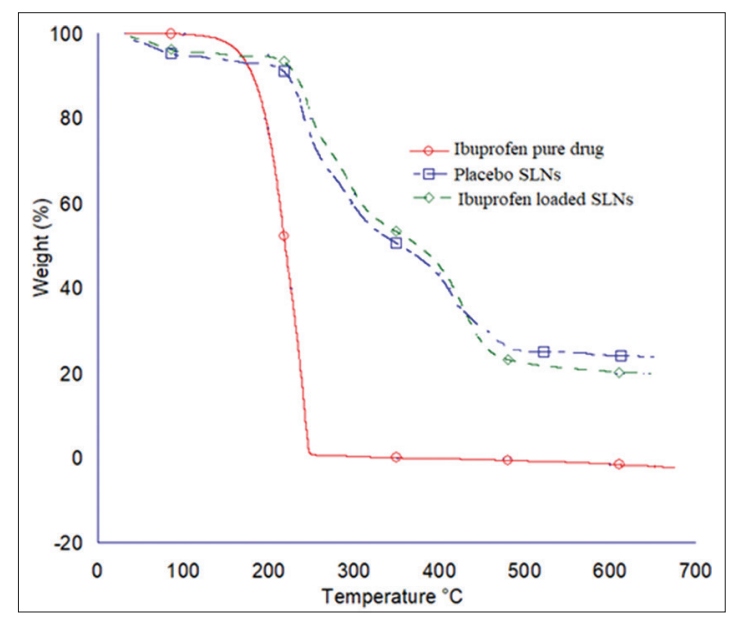

Fig. 5: Thermogravimetric analysis thermograms for ibuprofen (IBU)-loaded solid lipid nanoparticles (SLNs), placebo SLNs, and pure IBU drug

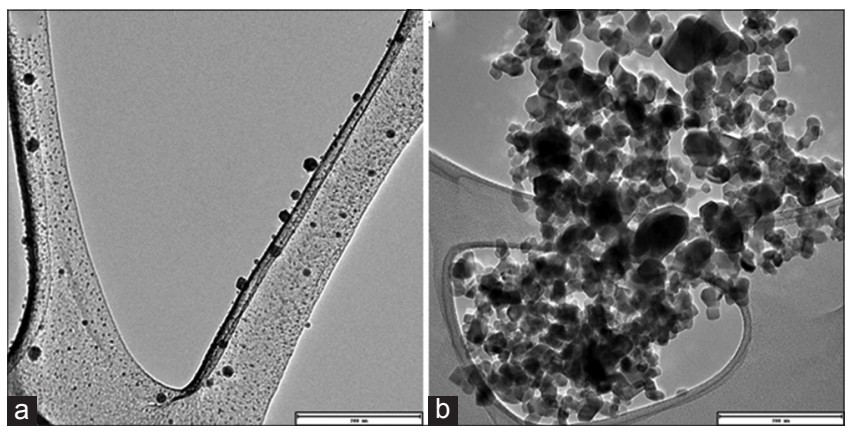

Fig. 6: Transmission electron microscopy images of placebo nanoparticles (a) and ibuprofen-loaded nanoparticles (b) 


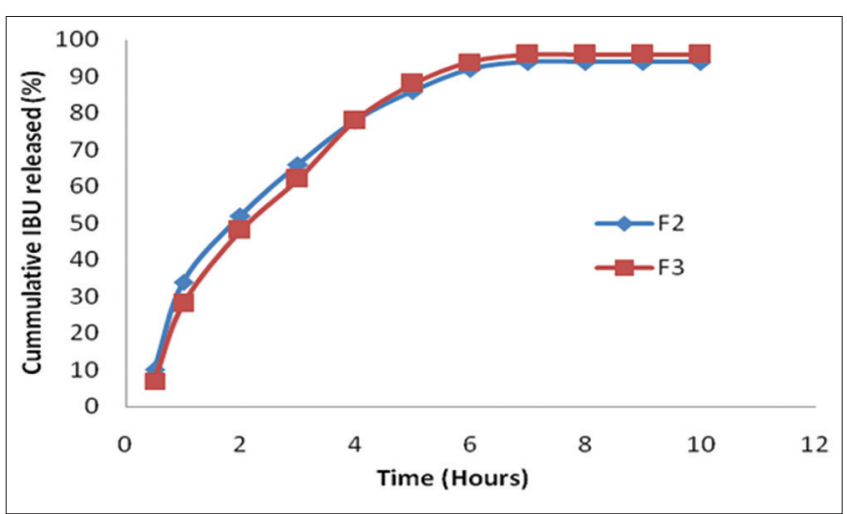

Fig. 7: Comparative cumulative percentage in vitro release of for ibuprofen-loaded solid lipid nanoparticles

the slightly larger particle sizes and lower DL capacity. Similar results were reported by Begum and Krisha [22], where they prepared IBUloaded polymeric nanoparticles with different stabilizers and varied the organic: aqueous ratio. The study reported a longer release profile for a higher ratio which was attributed to the higher entrapment efficiency of the formulation and partly due to the nature and quantity of the stabilizer [22]. In the current study, the entrapment efficiency is high coupled with a low DL capacity making the drug to be released slowly over a period of time $[17,19]$. PVP being a hydrophilic and swellable polymer, and it is present on the nanoparticle surface which forms a gel barrier for the release of the drug resulting in a sustained and controlled release in the formulations $[22,29,30]$.

\section{In vitro drug release kinetics}

To establish the mathematical model of drug release, the experimental data are fitted to different kinetic models. Various mathematical kinetic models such as zero-order, first-order, Higuchi, Korsmeyer-Peppas, and Hixson-Crowell models are used among others to justify the mechanism of drug release [20].

According to the principles of thermodynamics, the zero-order kinetic can be represented by the following equation $[20,29,30]$.

$\mathrm{C}_{\mathrm{t}}=\mathrm{C}_{0}+\mathrm{K}_{0} \mathrm{t}$

$C_{t}$ is the amount of drug released at time $t, C_{0}$ is the initial concentration of drug at time $\mathrm{t}=0, \mathrm{~K}_{0}$ is the zero-order rate constant.

A system is defined to be of zero-order kinetics if the process is of constant drug release from the matrix and is independent of the drug concentration. Hence, to study the drug release kinetics data obtained from in vitro drug release, the obtained data are plotted against time (cumulative drug release vs. time).

The slope of the above plot indicates the zero-order rate constant and the correlation coefficient of the above plot will give the information whether the drug release follows zero-order kinetics or not $[29,30]$. This model was thus applied to the release profile of IBU-loaded SLNs, and evaluation was done in the graphical presentation (Fig. 8). The obtained analysis indicates that drug release of IBU from the matrix system does not follow the principle of zero-order release kinetics due to the lower values of $\mathrm{R}^{2}\left(\mathrm{R}^{2}=0.764\right.$ for formulation $\mathrm{F} 2$ and $\mathrm{R}^{2}=0.0 .853$ for formulation F3).

The drug release which follows first-order kinetics can be represented by the equation [29-31].

$\log \mathrm{C}=\log \mathrm{C}_{0}-\mathrm{K} 1 \mathrm{t} / 2.303$.

$\mathrm{K}_{1}$ is the $1^{\text {st }}$ order rate equation.

$\mathrm{C}_{0}$ is the initial concentration of the drug.

$\mathrm{C}$ is the percentage of the drug remaining at time $\mathrm{t}$.

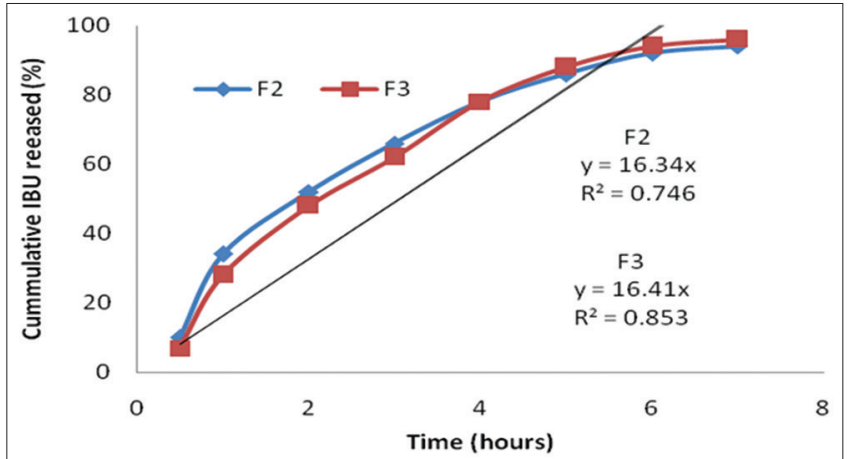

Fig. 8: Zero-order kinetic release for ibuprofen-loaded solid lipid nanoparticles

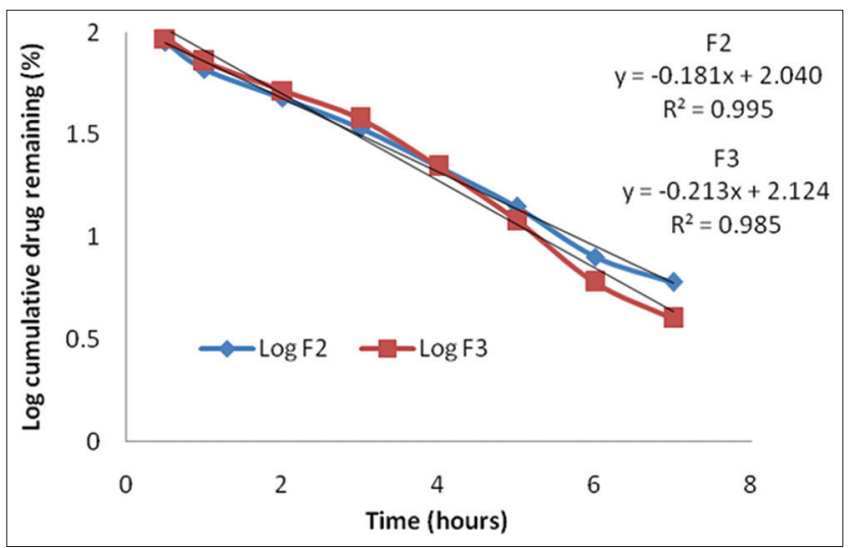

Fig. 9: First-order kinetic release of ibuprofen-loaded solid lipid nanoparticles

The drug release kinetics can be studied by obtaining data from the in vitro release and plotting the same against time. The graph will be of the log percentage of drug remaining versus time where the slope of the plot gives the first-order rate constant. The correlation coefficient of the plot will provide information that will be useful to conclude whether the release obeys first-order kinetics [30,31]. This model was thus applied in the release profile of IBU-loaded SLNs and the evaluation graphically represented in Fig. 9.

From the data obtained, it is evident that IBU release from both formulations (F2 and F3) from the matrix follows the principle of firstorder release kinetics and indicative of the higher values of the coefficient of correlation ( $\mathrm{R}^{2}$ of 0.995 and 0.985 , respectively). This technically means that the IBU release from the matrix system of the nanoparticles is dependent on the concentration present within the nanoparticles.

Higuchi developed several theoretical models to describe the release rate of water-soluble and poorly soluble drugs from matrix systems [32]. Several studies have used the Higuchi model to interpret experimental drug release from solid dispersions $[20,33$,$] . The release$ experimental data obtained were fitted into the Higuchi model, and the graph is shown in Fig. 10.

The results indicate that the two formulations best fitted the Higuchi square root model and followed Higuchi drug release kinetics as the plots show high linearity with a correlation coefficient $\left(R^{2}\right)$ higher than 0.95 [25]. This typically indicates that the release of the drug from the matrix system is diffusion controlled [20]. Formulations containing about $20 \%$ DL have been reported to release for a longer period of time and has usually obeyed the Higuchi square root model [19,20,31].

Korsmeyer developed a semi-empirical model to describe drug release for polymeric systems and summarized it in the equation below $[34,35]$. 
$\frac{M_{t}}{M_{\alpha}}=K t^{n}$

Where $\mathrm{K}$ is a constant incorporating structural and geometrical characteristics of the system, $M_{t} / M_{\alpha}$ is the fraction release of the drug at time $t$ and $n$ is the release exponent indicative of drug release mechanism. Peppas then characterized different release mechanisms using this $n$ value and his conclusion is provided in Table $3[22,34,35]$.

In the current study, the diffusion mechanism of the drug release was confirmed by Korsmeyer-Peppas plots with $\mathrm{R}^{2}$ values 0.968 and 0.972 ,

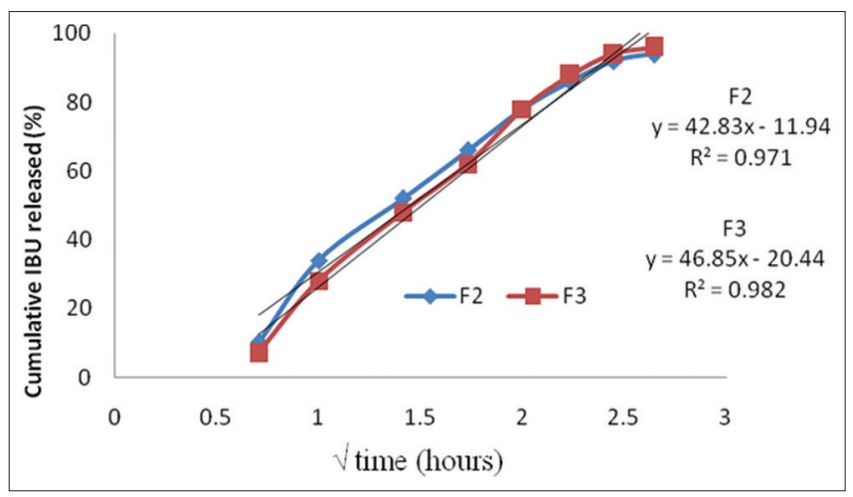

Fig. 10: Higuchi square root model indicating solid lipid nanoparticles for F2 and F3

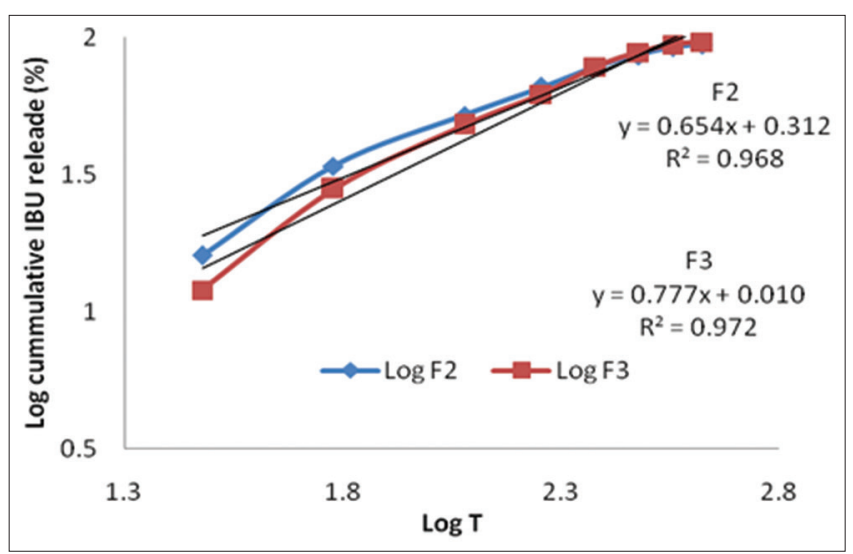

Fig. 11: Korsmeyer-Peppas plots indicating ibuprofen-loaded solid lipid nanoparticles respectively, and the gradient values (n) 0.654 and 0.777 (Fig. 11 and Table 4) which fall between $0.42<\mathrm{n}<0.85$ (Table 3 ) as our nanoparticles are spherical from the TEM results.

The values of the slopes indicate that the IBU release mechanisms from the nanoparticles followed an anomalous transport or non-Fickian diffusion transport [34-37]. This means that the diffusion in time dependent and the release of the drug from the matrix and polymer is dependent on the concentration of the drug thus supports the firstorder reaction theory [34-38]. Similar results were obtained where IBU was loaded into ethyl cellulose nanoparticles by nanoprecipitation technique [22]. The study reported nanoparticles prepared with tween 80 and tween 20 as the surfactant to have an $n=0.3$ and 0.4 , respectively, indicating they followed a Fickian diffusion while those prepared by PVA as the surfactant had an $\mathrm{n}=0.5$ indicating they were of non-Fickian diffusion [22].

\section{CONCLUSION}

IBU loaded SLNs were successfully prepared by single emulsion, solvent evaporation technique with SA as a matrix and PVP as the surfactant. There was a significant decrease in particle size with an increase in the internal to external ratio. The formulation with 1:6 ratios was preferred due to smaller particle size and high EE. The IBUloaded nanoparticles were found to be amorphous as compared to the crystalline nature of the IBU pure drug. Formulations F2 and F3 were found to sustain drug release for about $7 \mathrm{~h}$ and $8 \mathrm{~h}$, respectively, and they both followed first-order kinetics with a non-Fickian diffusion mechanism.

\section{ACKNOWLEDGMENTS}

The authors wish to acknowledge the Department of Chemistry, Vaal University of Technology for providing the laboratory space where the experiments and analysis were done. Much appreciation also goes to Mr. Samuel Olayinka Awofiranye and Ms. Samkeliso Takaidza, both from Vaal University of Technology for their profound assistance during the entire project. Dr. Pierre Kalenga of the University of Witwatersrand is also acknowledged for his assistance in characterization and reviewing of this article before submission.

\section{AUTHORS' CONTRIBUTIONS}

The authors declare that the work reported herein was performed by the two authors. Prof. Makwena Moloto designed the study, procured the necessary chemicals required, supervised the entire work and proofread the manuscript before submission. On the other hand, Dr. Nyaigoti Omwoyo performed the laboratory experiments and drafted the manuscript.

Table 3: Drug release mechanisms and diffusion exponent for polymeric controlled delivery systems of different geometries

\begin{tabular}{llll}
\hline Release exponent (n) & & Drug transport mechanism \\
\cline { 1 - 2 } Thin film & Cylinder & Sphere & \\
\hline$<0.5$ & $<0.44$ & $<0.42$ & Quasi Fickian \\
0.5 & 0.44 & 0.42 & Fickian diffusion \\
$0.5<\mathrm{n}<1.0$ & $0.45<\mathrm{n}<0.89$ & $0.42<\mathrm{n}<0.85$ & Non-Fickian diffusion/anomalous transport \\
1.0 & 0.89 & $0.85-1.0$ & Case II transport/non-Fickian case II \\
$>1.0$ & $>0.89$ & $>1.0$ & Non-Fickian super case II \\
\hline
\end{tabular}

Table 4: Correlation coefficient for different kinetic models

\begin{tabular}{|c|c|c|c|c|c|}
\hline \multirow[t]{2}{*}{ Formulation } & \multirow{2}{*}{$\begin{array}{l}\text { Zero-order } \\
\left(\mathrm{R}^{2}\right)\end{array}$} & \multirow{2}{*}{$\begin{array}{l}\text { First-order } \\
\left(\mathrm{R}^{2}\right)\end{array}$} & \multirow{2}{*}{$\begin{array}{l}\text { Higuchi } \\
\left(R^{2}\right)\end{array}$} & \multicolumn{2}{|c|}{ Korsmeyer-Peppas } \\
\hline & & & & $\left(\mathbf{R}^{2}\right)$ & (n) \\
\hline F2 & 0.746 & 0.995 & 0.971 & 0.968 & 0.654 \\
\hline F1 & 0.853 & 0.985 & 0.982 & 0.972 & 0.777 \\
\hline
\end{tabular}




\section{CONFLICTS OF INTEREST}

The authors declare that they have no conflicts of interest regarding the publication of this article.

\section{REFERENCES}

1. Yiyun C, Tongwen X. Dendrimers as potential drug carriers. Part I. Solubilization of non-steroidal anti-inflammatory drugs in the presence of polyamidoamine dendrimers. Eur J Med Chem 2005;40:1188-92.

2. Reis CP, Ferreira JP, Candeias S. Ibuprofen nanoparticles for oral delivery : Proof of concept. Biotherapeutic Discov 2013;4:1-5.

3. Jiang B, Hu L, Gao C, Shen J. Ibuprofen-loaded nanoparticles prepared by a co-precipitation method and their release properties. Int J Pharm 2005;304:220-30.

4. Zhu KJ, Li Y, Jiang HL, Yasuda H, Ichimaru A, Yamamoto K, et al. Preparation, characterization and in vitro release properties of ibuprofen-loaded microspheres based on polylactide, poly(epsiloncaprolactone) and their copolymers. J Microencapsul 2005;22:25-36.

5. Kumar S, Rajkumar S, Ruckmani K. Formulation and evaluation of ibuprofen loaded nanoparticles for improved anti-inflamatory activity. Acta pharm Turc 2003;45:125-30.

6. Potthast H, Dressman JB, Junginger HE, Midha KK, Oeser H, Shah VP, et al. Biowaiver monographs for immediate release solid oral dosage forms: Ibuprofen. J Pharm Sci 2005;94:2121-31.

7. Sweetman CS. Martindale: The Complete Drug Reference. Scriba GKE. Vol. 74. London, UK: Pharmaceuticl Press; 2011. p. 647-8.

8. Moore N. Ibuprofen: A journey from prescription to over-the-counter use. J R Soc Med 2007;100 Suppl 48:2-6.

9. Borhade N, Pathan AR, Halder S, Karwa M, Dhiman M, Pamidiboina V, et al. NO-NSAIDs. Part 3: Nitric oxide-releasing prodrugs of non-steroidal anti-inflammatory drugs. Chem Pharm Bull (Tokyo) 2012;60:465-81

10. Shimpi S, Chauhan B, Shimpi P. Cyclodextrins: Application in different routes of drug administration. Acta Pharm 2005;55:139-56.

11. Mansouri M, Pouretedal HR, Vosoughi V. Preparation and characterization of ibuprofen nanoparticles by using solvent/antisolvent precipitation. Open Conf Proc J 2011;2:88-94.

12. Nada A, Bandarkar F, Al-basarah Y. Formulation of ibuprofen nanoparticles. Asian J Pharm 2017;11:4-10.

13. Csóka G, Marton S, Zelko R, Otomo N, Antal I. Application of sucrose fatty acid esters in transdermal therapeutic systems. Eur J Pharm Biopharm 2007;65:233-7.

14. Mehnert W, Mäder K. Solid lipid nanoparticles: Production, characterization and applications. Adv Drug Deliv Rev 2001;47:165-96.

15. Sreelola V, Sailaja AK. Preparation and characterisation of ibuprofen loaded polymeric nanoparticles by solvent evaporation technique. Int $\mathrm{J}$ Pharm Pharm Sci 2014;6:6-11.

16. Jan SU, Khan GM, Hussain I. Formulation development and investigation of ibuprofen controlled release tablets with hydrophilic polymers and the effect of co-excipients on drug release patterns. Pak J Pharm Sci 2012;25:751-6.

17. Omwoyo WN, Ogutu B, Oloo F, Swai H, Kalombo L, Melariri P, et al. Preparation, characterization, and optimization of primaquine-loaded solid lipid nanoparticles. Int J Nanomedicine 2014;9:3865-74.

18. Potta SG, Minemi S, Nukala RK, Peinado C, Lamprou DA, Urquhart A, et al. Preparation and characterization of ibuprofen solid lipid nanoparticles with enhanced solubility. J Microencapsul 2011;28:74-81

19. Omwoyo WN, Melariri P, Gathirwa JW, Oloo F, Mahanga GM, Kalombo L, et al. Development, characterization and antimalarial efficacy of dihydroartemisinin loaded solid lipid nanoparticles. Nanomedicine 2016;12:801-9.

20. Giri TK, Kumar K, Alexander A, Ajazuddin AA, Badwaik H, Tripathi DK. A novel and alternative approach to controlled release drug delivery system based on solid dispersion technique. Bull Fac Pharm Cairo Univ 2012;50:147-59.

21. Westesen K, Bunjes H, Koch MH. Physicochemical characterization of lipid nanoparticles and evaluation of their drug loading capacity and sustained release potential. J Control Release 1997;48:223-36.

22. Begum N, Sailaja KA. Effect of formulation variables on the preparation of ibuprofen loaded polymeric nanoparticles. Pharm Nanotechnol 2016;3:111-21.

23. Cooper DL, Harirforoosh S. Design and optimization of PLGA-based diclofenac loaded nanoparticles. PLoS One 2014;9:e87326.

24. Akbari Z, Amanlou M, Karimi-Sabet J, Golestani A, Niassar MS. Production of ibuprofen-loaded solid lipid nanoparticles using rapid expansion of supercritical solution. J Nano Res 2015;31:15-29.

25. Sastre V, Ghaly ES. Controlled release ibuprofen nanoparticles : Physico-chemical characterization and drug release. Int J Pharm Pharm Sci 2014;6:99-107.

26. Hasnain MS, Nayak AK. Solubility and dissolution enhancement of ibuprofen by solid dispersion technique using PEG 6000-PVP K 30 combination carrier. Bulg J Sci Educ 2012;21:118-32.

27. Kotcherlakota R, Barui AK, Prashar S, Fajardo M, Briones D, Rodríguez-Diéguez A, et al. Curcumin loaded mesoporous silica: An effective drug delivery system for cancer treatment. Biomater Sci 2016;4:448-59.

28. Patil SJ, Kumar SY, Mokale JV, Naik BJ. Development of surfactant free nanoparticles by a single emulsion high pressure homogenization technique and effect of formulation parameters on the drug entrapment and release. Int J Pharm 2013;3:843-52.

29. Vineeth P, Rao PR, Kumar K, Babu BD, Veerabhadra RA, Suresh BK. Influence of organic solvents on nanoparticle formation and surfactants on release behaviour in-vitro using costunolide as model anticancer agent. Int J Pharm Pharm Sci 2014;6:638-45.

30. Gouda R, Baishya H, Quing Z. Application of mathematical models in drug release kinetics of carbidopa and levodopa ER tablets. J Dev Drugs 2017;6:1-8.

31. Dash S, Murthy PN, Nath L, Chowdhury P. Kinetic modeling on drug release from controlled drug delivery systems. Acta Pol Pharm 2010;67:217-23.

32. Higuchi T. Mechanism of sustained-action medication. theoretical analysis of rate of release of solid drugs dispersed in solid matrices. J Pharm Sci 1963;52:1145-9.

33. Owuor J, Oloo F, Ngetich J, Nyaigoti W, Gathirwa J. Comparison of freeze and spray drying to obtain primaquine-loaded solid lipid nanoparticles. Drug Des Dev Ther Comp 2017;1:1-8.

34. Ritger P, Peppas NA. A simple equation for description of solute release I. Fickian and non-fickian release from non-swellable devices in the form of slabs, spheres, cylinders or discs. J Control Release 1987;5:23-6.

35. Peppas NA, Khare AR. Preparation, structure and diffusional behavior of hydrogels in controlled release. Adv Drug Deliv Rev 1993;11:1-35.

36. Basak SC, Kumar KS, Ramalingam M. Design and release characteristics of sustained release tablet containing metformin $\mathrm{HCl}$. Rev Bras Cienc Farm J Pharm Sci 2008;44:477-83.

37. Jana U, Mohanty AK, Manna PK, Mohanta GP. Preparation and characterization of nebivolol nanoparticles using eudragit ${ }^{\circledR}$ RS100. Colloids Surf B Biointerfaces 2014;113:269-75

38. Arifin DY, Lee LY, Wang CH. Mathematical modeling and simulation of drug release from microspheres: Implications to drug delivery systems. Adv Drug Deliv Rev 2006;58:1274-325. 